\title{
Weighted O-Minimal Hybrid Systems Are More Decidable Than Weighted Timed Automata!*
}

\author{
Patricia Bouyer, Thomas Brihaye, and Fabrice Chevalier \\ LSV - CNRS \& ENS de Cachan \\ 61, avenue du Président Wilson, 94230 Cachan, France \\ \{bouyer, brihaye, chevalie\}@lsv.ens-cachan.fr
}

\begin{abstract}
We consider weighted o-minimal hybrid systems, which extend classical o-minimal hybrid systems with cost functions. These cost functions are "observer variables" which increase while the system evolves but do not constrain the behaviour of the system. In this paper, we prove two main results: $(i)$ optimal o-minimal hybrid games are decidable; $(i i)$ the model-checking of WCTL, an extension of CTL which can constrain the cost variables, is decidable over that model. This has to be compared with the same problems in the framework of timed automata where both problems are undecidable in general, while they are decidable for the restricted class of one-clock timed automata.
\end{abstract}

\section{Introduction}

O-minimal hybrid systems. Hybrid systems are finite-state machines where each state is equipped with a continuous dynamics. In the last thirty years, formal verification of such systems has become a very active field of research in computer science. In this context, hybrid automata, an extension of timed automata [AD94], have been intensively studied [Hen95,Hen96], and decidable subclasses of hybrid systems have been identified like initialized rectangular hybrid automata [Hen96] or o-minimal hybrid automata. This latter model has been pointed out in [LPS00] as an interesting class of systems with very rich continuous dynamics, but limited discrete steps (at each discrete step, all variables have to be reset, independently from their initial values). Behaviours of such a system can be decoupled into continuous and discrete parts, properties of a global o-minimal system can thus be deduced directly from properties of the continuous parts of the system. This property and properties of o-minimal structures (see [vdD98] for an overview) are exploited in the word encoding techniques, which have been developed in [BMRT04] for (finitely) abstracting behaviours of the system. Using techniques based on this abstraction, reachability properties [BM05], reachability control properties [BBC06] have been proved decidable for o-minimal hybrid systems. This technique was also used in order to compute a (tight) exponential bound on the size of the coarsest finite bisimulation of Pfaffian hybrid systems (see [KV06]).

\footnotetext{
* Work partly supported by ACI Sécurité Informatique CORTOS.
} 
Models for resource consumption. A research direction which has recently received substantial attention is the twist or extension of (decidable) models for representing more fairly interesting properties of embedded systems, for instance resource consumption. In that context, timed automata [AD94] have been extended with cost information bringing the model of weighted (or priced) timed automata $\left[\mathrm{ALP} 01, \mathrm{BFH}^{+} 01\right]$. A timed automaton is a finite automaton with clock variables (i.e. variables which increase as global time) that can be tested towards constants or reset. In the model of weighted timed automata, an extra cost variable is added, which is used as an observer variable (it does not constrain the behaviour of the system), evolving linearly while time elapses, and subject to discrete jumps when discrete transitions are taken. This model was appealing for expressing quantitative properties of real-time systems, which was concretized by the decidability of the optimal reachability problem (find the best way - in terms of cost - of reaching a given state) $\left[\mathrm{ALP} 01, \mathrm{BFH}^{+} 01, \mathrm{BBBR} 06\right]$ together with the development of the tool Uppaal Cora [cor06], and then by the computability of the optimal mean-cost (find the best way for the system to have a "cost per time unit" as low as possible) [BBL04]. However, more involved properties like cost-optimal reachability control (find the minimum cost that can be ensured for reaching a given state, whatever does the environment in which the system is embedded) or WCTL model-checking (WCTL extends the branching-time temporal logic CTL with cost constraints on modalities [BBR04,BBR06]) have been proved undecidable for weighted timed automata with three clocks or more, see [BBR04,BBR05,BBM06]. Though both problems have recently been proved decidable for one-clock weighted timed automata [BLMR06,BLM07] these undecidability results are nevertheless disappointing, because the one-clock assumption is rather restrictive.

Our contributions. In this paper, we propose a natural extension of o-minimal hybrid systems with (definable) positive cost functions which increase while time progresses and which can be used in an optimization criterion, as in the case of weighted timed automata. It is worth noticing here that though the underlying system is o-minimal, this extended model, called weighted o-minimal hybrid automaton, is not o-minimal as we do not require that the cost is reset when a discrete transition is taken. However, we prove in this paper that the cost-optimal reachability control problem and the WCTL model-checking problem are both decidable for this class of systems. Because of the existing results on weighted timed automata, this is really a surprise, and makes o-minimal hybrid systems an analyzable, though powerful model. The decidability results of course partly rely on the word encoding techniques that we mentioned earlier, but also require refinements and involved techniques, specific to each of the two problems.

\section{General Background}

Let $\mathcal{M}$ be a structure. In this paper when we say that some relation, subset or function is definable, we mean it is first-order definable in the sense of the structure $\mathcal{M}$. A general reference for first-order logic is [Hod97]. We denote by 
$\operatorname{Th}(\mathcal{M})$ the theory of $\mathcal{M}$. In the sequel we only consider structures $\mathcal{M}$ that are expansions of ordered groups containing two constant symbols, i.e. $\mathcal{M}=$ $\langle M,+, 0,1,<, \ldots\rangle$.

\subsection{O-Minimality}

Let us recall the definition of o-minimal structures [PS86] and give some examples of such structures. The reader interested in o-minimality should refer to [vdD98] for further results and an extensive bibliography on this subject. In the sequel of the paper we focus on o-minimal structures with a decidable theory in order to obtain decidability and computability results.

Definition 1. A totally ordered structure $\mathcal{M}=\langle M,<, \ldots\rangle$ is o-minimal if every definable subset of $M$ is a finite union of points and open intervals (possibly unbounded).

Example 1. Examples of o-minimal structures are the ordered group of rationals $\langle\mathbb{Q},<,+, 0,1\rangle$, the ordered field of reals $\langle\mathbb{R},<,+, \cdot, 0,1\rangle$, the field of reals with restricted pfaffian functions and the exponential function [Wil96].

\subsection{O-Minimal Dynamical Systems}

In this subsection we define the notion of o-minimal dynamical systems.

Definition 2 (O-minimal dynamical system). An o-minimal dynamical system is a pair $(\mathcal{M}, \gamma)$ where:

- $\mathcal{M}=\langle M,+, 0,1,<, \ldots\rangle$ is an o-minimal expansion of an ordered group,

$-\gamma: V_{1} \times V \rightarrow V_{2}$ is a function definable in $\mathcal{M}$ (where $V_{1} \subseteq M^{k_{1}}, V \subseteq M$, and $\left.V_{2} \subseteq M^{k_{2}}\right)$. ${ }^{1}$ The function $\gamma$ is called the dynamics of the system.

Classically, when $M$ is the set of the real numbers, we see $V$ as the time, $V_{1} \times V$ as the space-time, $V_{2}$ as the (output) space and $V_{1}$ as the input space. We keep this terminology in the more general context of a structure $\mathcal{M}$.

Example 2. We can view the continuous dynamics of timed automata [AD94] as an o-minimal dynamical system. In this case, we have that $\mathcal{M}=\langle\mathbb{R},<,+, 0,1\rangle$ and the dynamics $\gamma:\left(\mathbb{R}^{+}\right)^{n} \times \mathbb{R}^{+} \rightarrow\left(\mathbb{R}^{+}\right)^{n}$ is defined by $\gamma\left(x_{1}, \ldots, x_{n}, t\right)=$ $\left(x_{1}+t, \ldots, x_{n}+t\right)$.

We define a transition system associated with the dynamical system, this definition is an adaptation to our context of the classical continuous transition system in the case of hybrid systems (see [LPS00] for example).

Definition 3. Given $(\mathcal{M}, \gamma)$ a dynamical system, we define a transition system $T_{\gamma}=\left(Q, \Sigma, \rightarrow_{\gamma}\right)$ associated with $(\mathcal{M}, \gamma)$ by: the set $Q$ of states is $V_{2}$; the set $\Sigma$ of events is $M^{+}=\{m \in M \mid m \geq 0\}$; the transition relation $y_{1} \stackrel{\tau}{\rightarrow}_{\gamma} y_{2}$ is defined by: $\exists x \in V_{1}, \exists t_{1}, t_{2}, \in V$ s.t. $t_{1} \leq t_{2}, \gamma\left(x, t_{1}\right)=y_{1}, \gamma\left(x, t_{2}\right)=y_{2}$ and $\tau=t_{2}-t_{1}$.

\footnotetext{
${ }^{1}$ We use these notations in the rest of the paper.
} 


\subsection{O-Minimal Hybrid Systems and Games}

In this subsection, we define o-minimal hybrid systems and games

Definition 4 (O-minimal hybrid systems). Let $\mathcal{M}=(M,+, 0,1,<, \cdots)$ be an o-minimal structure. An $\mathcal{M}$-hybrid system (or simply o-minimal hybrid system) $\mathcal{H}$ is a tuple $(Q, \Sigma, \delta, \gamma)$ where $Q$ is a finite set of locations, $\Sigma$ is a finite set of actions, $\delta$ consists in a finite number of transitions $\left(q, g, a, R, q^{\prime}\right) \in$ $Q \times 2^{V_{2}} \times \Sigma \times 2^{V_{2}} \times Q$ where the guard $g$ and the reset $R$ are definable in $\mathcal{M}$, and $\gamma$ maps every location $q \in Q$ to a dynamic $\gamma_{q}: V_{1} \times V \rightarrow V_{2}$ definable in $\mathcal{M}$.

An $\mathcal{M}$-hybrid system $\mathcal{H}=(Q, \Sigma, \delta, \gamma)$ defines a mixed transition system $T_{\mathcal{H}}=(S, \Gamma, \rightarrow)$ where:

- the set $S$ of states is $Q \times V_{2}$;

- the set $\Gamma$ of labels is $M^{+} \cup \Sigma$;

- the transition relation $(q, y) \stackrel{e}{\rightarrow}\left(q^{\prime}, y^{\prime}\right)$ is defined when:

- $e \in \Sigma$ and there exists $\left(q, g, e, R, q^{\prime}\right) \in \delta$ with $y \in g$ and $y^{\prime} \in R(y)$, or

- $e \in M^{+}, q=q^{\prime}$, and $y \stackrel{e}{\rightarrow} \gamma_{q} y^{\prime}$ where $\gamma_{q}$ is the dynamic in location $q$.

We will also need more precise notions of transitions. When $(q, y) \stackrel{\tau}{\rightarrow}\left(q, y^{\prime}\right)$ with $\tau \in M^{+}$, this is due to some choice of $(x, t) \in V_{1} \times V$ such that $\gamma_{q}(x, t)=y$. We say that $(q, x, t, y) \stackrel{\tau}{\rightarrow}\left(q^{\prime}, x^{\prime}, t^{\prime}, y^{\prime}\right)$ if $q=q^{\prime}, x=x^{\prime}, t^{\prime}=t+\tau, \gamma_{q}(x, t)=y$ and $\gamma_{q}^{\prime}\left(x^{\prime}, t^{\prime}\right)=y^{\prime}$. We say that an action $(\tau, a) \in M^{+} \times \Sigma$ is enabled in a state $(q, x, t, y)$ if there exists $\left(q^{\prime}, x^{\prime}, t^{\prime}, y^{\prime}\right)$ and $\left(q^{\prime \prime}, x^{\prime \prime}, t^{\prime \prime}, y^{\prime \prime}\right)$ such that $(q, x, t, y) \stackrel{\tau}{\rightarrow}$ $\left(q^{\prime}, x^{\prime}, t^{\prime}, y^{\prime}\right) \stackrel{a}{\rightarrow}\left(q^{\prime \prime}, x^{\prime \prime}, t^{\prime \prime}, y^{\prime \prime}\right)$. We then write $(q, x, t, y) \stackrel{\tau, a}{\longrightarrow}\left(q^{\prime \prime}, x^{\prime \prime}, t^{\prime \prime}, y^{\prime \prime}\right)$. We note $\operatorname{Enb}(q, x, t, y)$ the set of actions enabled in $(q, x, t, y)$.

A run of $\mathcal{H}$ is a(n) (in)finite sequence $\varrho=\left(q_{0}, x_{0}, t_{0}, y_{0}\right) \stackrel{\tau_{1}, a_{1}}{\longrightarrow}\left(q_{1}, x_{1}, t_{1}, y_{1}\right) \cdots$ A position $\pi$ along $\varrho$ is a pair $(i, \tau) \in \mathbb{N} \times M^{+}$such that $\tau \leq \tau_{i+1}$. We define $\varrho[(i, \tau)]=\left(q_{i}, \gamma_{q_{i}}\left(x_{i}, t_{i}+\tau\right)\right)$. Let us notice that the positions of a given run are totally ordered in a natural way. If $\varrho$ is finite we define $\operatorname{last}(\varrho)=\left(q_{n}, x_{n}, t_{n}, y_{n}\right)$. We note $\operatorname{Runs}_{f}(\mathcal{H})$ the set of finite runs in $\mathcal{H}$.

An interesting tool to study hybrid systems is the (time-abstract) bisimulation $^{2}$ (see [Hen95]). One of the main results concerning o-minimal hybrid systems is that they admit finite bisimulation quotient. This result has been first proved in [LPS00], it was reproved in [Dav99] in a more topological way, amongst a lot of other interesting results. In [Bri06a], the existence of finite bisimulations for o-minimal hybrid systems is proved by means of the suffix partition, a technique initiated in [BMRT04,BM05,Bri06b].

Theorem 5. ([Bri06a, Theorem 12.6.14])

Let $\mathcal{H}$ be an o-minimal hybrid system and $\mathcal{P}_{\mathcal{H}}$ be a finite and definable partition of $Q \times V_{2}$ respecting the guards and the resets of $\mathcal{H}$. There is a finite and definable partition, noted $\operatorname{Suf}\left(\mathcal{P}_{\mathcal{H}}\right)$, inducing a bisimulation on $\mathcal{H}$.

${ }^{2}$ Two systems are time-abstract bisimilar whenever they can both do the same actions and wait some delay. 
O-minimal hybrid systems are models for closed systems, where all transitions are controlled. If we want to consider open systems where we distinguish between the actions of a controller and of an environment, we need to consider games on o-minimal hybrid systems. We are now going to define this notion.

Definition 6 (O-minimal game). Let $\mathcal{M}=(M,+, 0,1,<, \cdots)$ be an o-minimal structure. An $\mathcal{M}$-game (or simply an o-minimal game) is a tuple ( $Q$, Goal, $\Sigma, \delta, \gamma)$ where Goal $\subseteq Q$ is a subset of winning locations, $(Q, \Sigma, \delta, \gamma)$ is an $\mathcal{M}$-hybrid system and $\Sigma$ is partitioned into two subsets $\Sigma_{c}$ and $\Sigma_{u}$ corresponding to controllable and uncontrollable actions.

Let $\mathcal{H}$ be an o-minimal game. The game is played by two players, the controller and the environment; the goal of the controller is to reach a winning state whatever the environment does. In every state $s$, the controller picks a delay $\tau$ and an action $a \in \Sigma_{c}$ such that there is a transition $s \stackrel{\tau, a}{\longrightarrow} s^{\prime}$. The environment has two choices:

- either it waits $\tau$ and executes a transition $s \stackrel{\tau, a}{\longrightarrow} s^{\prime}$ proposed by the controller, - or it waits $\tau^{\prime}, 0 \leq \tau^{\prime} \leq \tau$, and executes a transition $s \stackrel{\tau^{\prime}, u}{\longrightarrow} s^{\prime \prime}$ with $u \in \Sigma_{u}$.

The game then evolves to a new state (according to the choice of the environment) and the two players proceed to play as before.

We will now formalize the semantic through the concept of strategy.

Definition 7 (Strategy). A (controller) strategy ${ }^{3}$ is a partial function $\lambda$ from $\operatorname{Runs}_{f}(\mathcal{H})$ to $M^{+} \times \Sigma_{c}$ such that for all runs $\varrho$ in $\operatorname{Runs}_{f}(\mathcal{H})$, if $\lambda(\varrho)$ is defined, then $\lambda(\varrho)$ is enabled in last $(\varrho)$.

Intuitively, the strategy tells what needs to be done for controlling the system: at each instant it tells how much time we need to wait and which controllable action needs to be done after this delay. Note that even when the environment follows the controller's choice, it has to choose between several edges, each one labeled by the action given by the strategy (because the original game is not supposed to be deterministic).

Let $\varrho=\left(q_{0}, x_{0}, t_{0}^{\prime}, y_{0}\right) \stackrel{\tau_{1}, a_{1}}{\longrightarrow} \ldots$ be a run, and set for every $i, \varrho_{i}$ the prefix of $\varrho$ ending at position $(i, 0)$. The run $\varrho$ is said to be consistent with a strategy $\lambda$ when for all $i$, if $\lambda\left(\varrho_{i}\right)=(\tau, a)$ then either $\tau_{i+1}=\tau$ and $a_{i+1}=a$, or $\tau_{i+1} \leq \tau$ and $a_{i+1} \in \Sigma_{u}$. We denote by Outcome $(s, \lambda)$ the set of runs starting from a state $s$ of the (output) space consistent with the strategy $\lambda$. A run $\varrho=\left(q_{0}, x_{0}, t_{0}, y_{0}\right) \stackrel{\tau_{1}, a_{1}}{\longrightarrow}$ $\ldots \stackrel{\tau_{p}, a_{p}}{\longrightarrow}\left(q_{p}, x_{p}, t_{p}, y_{p}\right)$ is said winning if $q_{i} \in$ Goal for some $i$. A run $\varrho$ is said maximal with respect to a strategy $\lambda$ if it is infinite or if $\lambda(\varrho)$ is not defined. A strategy $\lambda$ is winning from a state $(q, y)$ if for all $(x, t)$ such that $\gamma(x, t)=y$, all maximal runs starting in $(q, x, t, y)$ compatible with $\lambda$ are winning.

\footnotetext{
${ }^{3}$ In the context of control problems, a strategy is also called a controller.
} 


\section{Weighted O-Minimal Hybrid Systems and Games}

In this section, we define the weighted o-minimal hybrid systems and games, which extend the two models of the previous section with cost functions. These cost functions give a quantitative information on the behaviours of the systems, which allows to give a measure of the performance of the system. These models are respectively inspired by the model of weighted (resp. priced) timed automata $\left[\mathrm{ALP} 01, \mathrm{BFH}^{+} 01\right]$ and the model of weighted (resp. priced) timed games [ABM04,BCFL04].

\subsection{Definitions}

We consider cost functions which are non-negative and time-non-decreasing. Note that cost functions in weighted timed automata [ALP01, $\mathrm{BFH}^{+} 01$ ] satisfy these hypotheses.

A non-negative and time-non-decreasing cost function is a definable function Cost : $Q \times V_{1} \times V \times M^{+} \rightarrow M^{+}$such that for all $q \in Q, x \in V_{1}, t \in V$ and $\tau_{1}, \tau_{2} \in M^{+}$with $\tau_{1} \leq \tau_{2}$ we have that $\operatorname{Cost}\left(q, x, t, \tau_{1}\right) \leq \operatorname{Cost}\left(q, x, t, \tau_{2}\right)$.

Definition 8 (Weighted o-minimal hybrid system and game). An $\mathcal{M}$ weighted hybrid system (resp. game) is an $\mathcal{M}$-hybrid system (resp. game) $\mathcal{H}=$ $(Q, \Sigma, \delta, \gamma)$ with a definable non-negative and time-non-decreasing cost function Cost.

The semantics of an o-minimal weighted hybrid system (resp. game) is the one of the underlying o-minimal hybrid system (resp. game). Hence, the cost function does not affect the behaviours of the system; it gives for every single step of the system a non-negative value, which represents the cost of evolving following that step. It naturally extends to a run in the system: let $\varrho=$

$\left(q_{0}, x_{0}, t_{0}, y_{0}\right) \stackrel{\tau_{1}, a_{1}}{\longrightarrow} \ldots \stackrel{\tau_{p}, a_{p}}{\longrightarrow}\left(q_{p}, x_{p}, t_{p}, y_{p}\right)$ be a finite run in $\mathcal{H}$. The cost of $\varrho$ is $\operatorname{Cost}(\varrho)=\sum_{i=1}^{p} \operatorname{Cost}\left(q_{i-1}, x_{i-1}, t_{i-1}, \tau_{i}\right)$.

Let us give an example of weighted o-minimal hybrid system.

Example 3 ([BLM07]). The weighted o-minimal hybrid system of Figure 1 models a never-ending process of repairing problems. The repair of a problem has a certain cost, captured in the model by the cost function Cost. As soon as a problem occurs (modeled by the $p b$ transition) the value of the cost grows with rate 1 , until actual repair is taking place by one of the transitions $r p_{1}$ (cheap but long repair) or $r p_{2}$ (expensive but quick repair). At most 24 time units after the occurrence of a problem it will have been repaired one way or another.

\subsection{Related Problems and Results}

In this subsection we define the two problems we are interested in: the costoptimal control problem and the WCTL model-checking problem. 


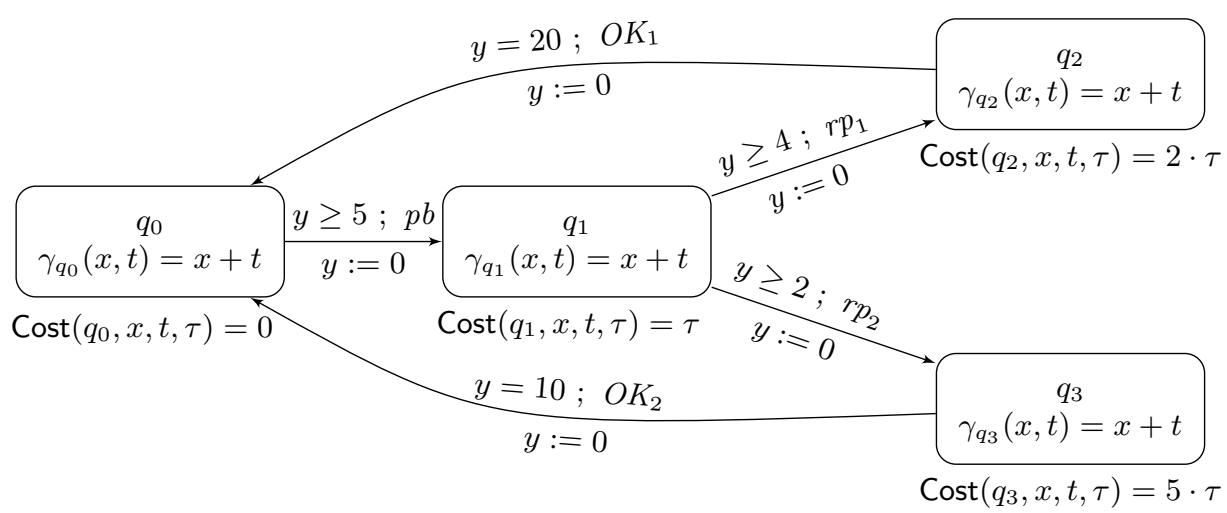

Fig. 1. A repair problem example

The cost-optimal control problem. The cost-optimal reachability control problem was first considered on weighted timed automata in [ABM04,BCFL04]. However it has been shown in [BBR05,BBM06] that the cost-optimal reachability control problem for weighted timed automata is undecidable.

In our context of weighted o-minimal games, the cost-optimal control problem asks what is the optimal cost for the controller to reach Goal whatever the environement does. In order to take the cost function into account, we now need to define the cost of a strategy from a state and the optimal cost from a state.

Definition 9 (Cost of a strategy from a state). Let ( $\mathcal{H}$, Cost) be a weighted o-minimal game, Goal be a subset of winning locations, $s$ be a state of the (output) space $V_{2}$ and $\lambda$ be a strategy. The $\operatorname{cost} \operatorname{Cost}(s, \lambda)$ of $\lambda$ from $s$ is defined by:

$$
\operatorname{Cost}(s, \lambda)=\sup \{\operatorname{Cost}(\varrho) \mid \varrho \in \operatorname{Outcome}(s, \lambda)\} .
$$

Intuitively the presence of the supremum is explained by the fact that the environment tries to maximize the cost.

Definition 10 (Optimal cost from a state). Let ( $\mathcal{H}$, Cost) be a weighted o-minimal game, Goal be a subset of winning locations and $s$ be a state of the (output) space $V_{2}$. The optimal cost $\operatorname{Opt} \operatorname{Cost}(q)$ associated with $s$ is defined by:

$$
\operatorname{Opt} \operatorname{Cost}(s)=\inf \{\operatorname{Cost}(s, \lambda) \mid \lambda \text { is a winning strategy }\} .
$$

A winning strategy from $s$ is called optimal whenever $\operatorname{Cost}(s, \lambda)=\operatorname{OptCost}(s)$.

Problem 1 (Cost-optimal control problem). Given a weighted o-minimal game $\mathcal{H}$, a definable state $s$ and a definable constant $c$, decide if there exists a winning strategy $\lambda$ from $q$ such that $\operatorname{Cost}(s, \lambda) \leq c$.

Problem 2 (Computation of the optimal cost). Given a weighted o-minimal game $\mathcal{H}$ and a definable state $s$, compute the optimal cost OptCost $(s)$. 
Remark 1. There is an optimal winning strategy from state $s$ iff the infimum can be replaced by a minimum in the definition of $\operatorname{OptCost}(s)$. If we solve problem 1 and 2, we can also determine if there is an optimal winning strategy by asking if there is a strategy $\lambda$ with $\operatorname{Cost}(s, \lambda) \leq \operatorname{OptCost}(s)$. In [BBR05,BBM06], it has been shown that the variant of Problem 1 for weighted timed automata is undecidable.

The WCTL model-checking problem. The logic WCTL ${ }^{4}$ has been proposed in the context of (weighted) timed systems as an extension of CTL with cost constraints on modalities [BBR04,BBM06,BLM07]. In our context, we define for every structure $\mathcal{M}$ the logic $\mathrm{WCTL}_{\mathcal{M}}$ over $\Sigma$ inductively as follows:

$$
\mathrm{WCTL}_{\mathcal{M}} \ni \varphi::=a|\varphi \vee \varphi| \neg \varphi\left|\mathrm{E} \varphi \mathrm{U}_{\sim_{c}} \varphi\right| \mathrm{A} \varphi \mathrm{U}_{\sim_{c}} \varphi
$$

where $a \in \Sigma, \sim \in\{<, \leq,=, \geq,>\}$ and $c$ is an $\mathcal{M}$-definable constant.

Let $\left(\mathcal{H}\right.$, Cost) be an $\mathcal{M}$-hybrid system. The semantics of $\mathrm{WCTL}_{\mathcal{M}}$ is defined for every state $(q, y) \in Q \times V_{2}$ of $(\mathcal{H}$, Cost $)$ as follows:

$$
\begin{aligned}
(q, y) \models a \Leftrightarrow & (q, y) \stackrel{a}{\rightarrow}\left(q^{\prime}, y^{\prime}\right) \text { for some }\left(q^{\prime}, y^{\prime}\right) \in Q \times V_{2}{ }^{5} \\
(q, y) \models \neg \varphi \Leftrightarrow & (q, y) \not \models \varphi \\
(q, y) \models \varphi_{1} \vee \varphi_{2} \Leftrightarrow & (q, y) \models \varphi_{1} \text { or }(q, y) \models \varphi_{2} \\
(q, y) \models \mathrm{E} \varphi_{1} \mathrm{U}_{\sim_{c} \varphi_{2} \Leftrightarrow} \Leftrightarrow & \text { there is an infinite run } \varrho \text { in } \mathcal{H} \text { from }(q, y) \\
& \text { s.t. } \varrho \models \varphi_{1} \mathrm{U}_{\sim_{c}} \varphi_{2} \\
(q, y) \models \mathrm{A} \varphi_{1} \mathrm{U}_{\sim_{c} \varphi_{2} \Leftrightarrow} \Leftrightarrow & \text { every infinite run } \varrho \text { in } \mathcal{H} \text { from }(q, y) \\
& \text { satisfies } \varrho \models \varphi_{1} \mathrm{U}_{\sim_{c} \varphi_{2}} \\
\varrho \models \varphi_{1} \mathrm{U}_{\sim_{c} \varphi_{2} \Leftrightarrow} \Leftrightarrow & \text { there exists } \pi \geq \text { position along } \varrho \text { s.t. } \\
& \varrho[\pi] \models \varphi_{2}, \text { for all positions } 0 \leq \pi^{\prime}<\pi \text { on } \varrho, \\
& \varrho\left[\pi^{\prime}\right] \models \varphi_{1} \vee \varphi_{2}, \text { and } \operatorname{Cost}(\varrho \leq \pi) \sim c^{6}
\end{aligned}
$$

We use true for $a \vee \neg a$, and classical "eventually" and "always" operators: $\mathrm{EF}_{\sim_{c} \phi} \phi$ (resp. $\left.\mathrm{AF}_{\sim_{c}} \phi\right)$ stands for $\mathrm{EtrueU}_{\sim_{c} \phi}$ (resp. A trueU $\mathrm{U}_{\sim_{c}} \phi$ ) and $\mathrm{AG}_{\sim_{c}} \phi$ (resp. $\mathrm{EG}_{\sim c} \phi$ ) stands for $\neg \mathrm{E} \mathrm{F}_{\sim c} \neg \phi$ (resp. $\neg \mathrm{AF}_{\sim c} \neg \phi$ ).

Let us give an example of WCTL formulae on the repair problem of Example 3.

Example 4. [BLM07] An example of a property that can be expressed with WCTL is "Whenever a problem occurs it can be repaired within a total cost of 55". It can be expressed with the following formula:

$$
\mathrm{AG}\left(p b \Longrightarrow \mathrm{EF}_{\leq 55}\left(O K_{1} \vee O K_{2}\right)\right) \text {. }
$$

One can easily check that this formula holds for every state of the weighted o-minimal hybrid system of Figure 1.

\footnotetext{
${ }^{4}$ WCTL stands for "Weighted CTL."

5 This can be viewed equivalently as atomic propositions.

${ }^{6}$ Following [Ras99] we use $\varphi_{1} \vee \varphi_{2}$ to handle open intervals in timed models.
} 
Problem 3 (Model-checking of WCTL). Given ( $\mathcal{H}$, Cost) an $\mathcal{M}$-weighted hybrid system, $\varphi$ a WCTL $\mathcal{W}_{\mathcal{M}}$-formula and $(q, y) \in Q \times V_{2}$ a definable state of $\mathcal{H}$, decide whether $(q, y) \models \varphi$.

Remark 2. Note that classical results on o-minimal hybrid systems cannot be used to solve the two problems presented above (for instance, weighted o-minimal hybrid systems do not have a finite bisimulation), and hence, ad-hoc proofs have to be developed.

\section{Solving the Cost-Optimal Control Problem}

In this section we prove the decidability of Problem 1.

Definition 11. Let $(\mathcal{H}$, Cost) be a weighted o-minimal game. We say that a run $\left(q_{0}, x_{0}, t_{0}, y_{0}\right) \stackrel{\tau_{1}, a_{1}}{\longrightarrow} \ldots$ crosses the transition $e=\left(q, g, a, R, q^{\prime}\right)$ at step $i$ if $q=q_{i-1}, q^{\prime}=q_{i}, a=a_{i}, \gamma_{q_{i-1}}\left(x_{i-1}, t_{i-1}+\tau_{i}\right) \in g$ and $y_{i} \in R$.

We are now going to prove a key proposition: we can restrict to winning strategies that cross each edge at most once. In order to prove this proposition we use the fact that the cost functions have non-negative values.

Proposition 12. Let $\mathcal{H}$ be a weighted o-minimal game and $(q, y)$ a state of $\mathcal{H}$. If there exists a winning strategy $\lambda$ from $(q, y)$ then there exists a winning strategy $\lambda_{\text {new }}$ with $\operatorname{Cost}\left((q, y), \lambda_{\text {new }}\right) \leq \operatorname{Cost}((q, y), \lambda)$ such that every run starting in $(q, y)$ compatible with $\lambda_{\text {new }}$ crosses each transition at most once.

Proof (Sketch). The idea of the proof is the following: for each transition $e$, we iteratively ensure that there is a winning strategy (of cost $c^{\prime} \leq c$ ) crossing $e$ at most once. See Outcome $((q, y), \lambda)$ as a(n infinitely branching) tree: every path in this tree is finite and reaches Goal, but it may cross $e$ several times. We construct a new strategy $\lambda_{\text {new }}$ which short-cuts $\lambda$ in the following sense: it simulates $\lambda$ until $e$ is crossed for the first time and then switches to a descendant in the tree from which all paths do not cross $e$ anymore (this is possible as $\lambda$ is winning). Such a strategy crosses $e$ at most once, its cost is smaller than that of $\lambda$ as its compatible runs are "shorter" than ones of $\lambda$ and the cost function is non-negative.

We now give a backward algorithm which computes the optimal cost, based on the formulation of the problem given in [LMM02,ABM04]. The termination of the algorithm relies on Proposition 12. For this, given $(q, y) \in Q \times V_{2}$ and $n \in \mathbb{N}$ we define $c_{n}(q, y)$, the optimal cost of reaching Goal from $(q, y)$ in at most $n$ steps. Formally we have that:

$$
\begin{aligned}
& -c_{0}(q, y)=0 \text { if } q \in \text { Goal, }+\infty \text { if } q \notin \text { Goal. } \\
& -c_{n+1}(q, y)= \\
& \sup _{\gamma_{q}(x, t)=y(\tau, a) \in \operatorname{Enb}(q, x, t, y)} \max \left\{\begin{array}{l}
\operatorname{Cost}_{c_{n}}^{\tau, a}(q, x, t, y) \\
\sup \left\{\operatorname{Cost}_{c_{n}}^{\tau^{\prime}, u}(q, x, t, y) \mid\left(\tau^{\prime}, u\right) \in \operatorname{Enb}(q, x, t, y), \tau^{\prime} \leq \tau\right\}
\end{array}\right. \\
& \text { where }(\tau, e) \in \operatorname{Enb}(q, x, t, y) \text { iff } e \in \operatorname{Enb}\left(q, x, t+\tau, \gamma_{q}(x, t+\tau)\right) \text {, and } \\
& \operatorname{Cost}_{c}^{\tau, e}(q, x, t, y)=\operatorname{Cost}(q, x, t, y, \tau)+\sup \left\{c\left(q^{\prime}, y^{\prime}\right) \mid(q, x, t, y) \stackrel{\tau, a}{\longrightarrow}\left(q^{\prime}, x^{\prime}, t^{\prime}, y^{\prime}\right)\right\} \text {. }
\end{aligned}
$$


Definition 13. A strategy $\lambda$ is said $n$-bounded from $(q, y)$ if every run compatible with $\lambda$ starting from $(q, y)$ has length at most $n$.

Lemma 14. For every $\varepsilon>0$, for every $(q, y) \in Q \times V_{2}$ s.t. $c_{n}(q, y)<+\infty$, there exists a definable $n$-bounded strategy $\lambda$ from $(q, y)$ such that $\operatorname{Cost}((q, y), \lambda) \leq$ $c_{n}(q, y)+\varepsilon$.

Lemma 15. If $\lambda$ is an $n$-bounded strategy from $(q, y)$ then $\operatorname{Cost}((q, y), \lambda) \geq$ $c_{n}(q, y)$.

Theorem 16. Let $\mathcal{M}=\langle M,+, 0,1, \ldots\rangle$ be an o-minimal structure such that $\mathrm{Th}(\mathcal{M})$ is decidable. The cost-optimal control problem over $\mathcal{M}$-weighted hybrid systems is decidable.

Proof. Let $\mathcal{H}$ be a weighted o-minimal game and $s$ a state of $\mathcal{H}$. Lemmas 14 and 15 imply that $c_{k}(s)=\inf \{\operatorname{Cost}(s, \lambda) \mid \lambda$ is a $k$-bounded strategy strategy $\}$.

Let $n$ be the number of transitions of $\mathcal{H}$. Proposition 12 shows that for every winning strategy from $s$ there is an $(n+1)$-bounded winning strategy from $s$ with smallest cost. Thus $\operatorname{Opt} \operatorname{Cost}(s)=\inf \{\operatorname{Cost}(s, \lambda) \mid \lambda$ is an $(n+$ 1 )-bounded strategy $\}=c_{n+1}(s)$. Note that $c_{n+1}(s)$ is computable since $\operatorname{Th}(\mathcal{M})$ is decidable.

We can moreover decide if the optimal cost can be achieved by a strategy: by Proposition 12 it is sufficient to enumerate all $(n+1)$-bounded strategies using $\tau_{i}$ 's as parameters and check if the cost of one of them is equal to $c_{n+1}(s)$.

Remark 3. Let us notice that Theorem 16 encompasses the decidability of the time-bounded reachability problem considered in [Gen05]. Moreover, a consequence of Theorem 16 is the decidability of the (cost-)optimal reachability problem formulated in $\left[\mathrm{ALP} 01, \mathrm{BFH}^{+} 01\right]$ for weighted timed automata.

\section{Solving the WCTL Model-Checking Problem}

The aim of this section is to prove the following decidability theorem. The techniques that we will develop for proving this result are partly inspired by the recent decidability proof for WCTL over one-clock weighted timed automata [BLM07].

Theorem 17. Let $\mathcal{M}=\langle M,+, 0,1,<, \ldots\rangle$ be an o-minimal structure such that $\mathcal{M}$ is Archimedian ${ }^{7}$ and $\operatorname{Th}(\mathcal{M})$ is decidable. The model-checking of $\mathrm{WCTL}_{\mathcal{M}}$ over $\mathcal{M}$-weighted hybrid systems is decidable.

If $\mathcal{P}$ and $\mathcal{P}^{\prime}$ are two partitions, we write $\mathcal{P} \sqcap \mathcal{P}^{\prime}$ for the joint partition, i.e. the smallest partition that refines both $\mathcal{P}$ and $\mathcal{P}^{\prime}$. Let us notice that if $\mathcal{P}$ and $\mathcal{P}^{\prime}$ are both finite and definable the joint partition $\mathcal{P} \sqcap \mathcal{P}^{\prime}$ is also finite and definable.

Let $\mathcal{H}=(Q$, Goal, $\Sigma, \delta, \gamma)$ be an $\mathcal{M}$-weighted hybrid system. On each location $q \in Q$, let us denote by $\mathcal{P}_{q}$ the partition induced by the guards and the resets

\footnotetext{
$\overline{7}$ A structure is Archimedian whenever for every pair $(a, b) \in M^{2}$ such that $a \neq 0$, there exists some integer $n$ such that $n \cdot a>b$.
} 
associated with location $q$. We denote by $\mathcal{P}_{\mathcal{H}}$ the partition of state space $S$ $=Q \times V_{2}$ induced by the $\mathcal{P}_{q}$ 's. $\mathcal{P}_{\mathcal{H}}$ is a finite definable partition of $S$.

Two states of the same piece $P$ of $\mathcal{P}_{\mathcal{H}}$ agree on all atomic formulae $a \in \Sigma$. We will now inductively construct for every $\mathrm{WCTL}_{\mathcal{M}}$-formula $\varphi$ a refined (finite and definable) partition $\mathcal{P}_{\varphi}$ of $\mathcal{P}_{\mathcal{H}}$ such that two states of a piece of $\mathcal{P}_{\varphi}$ agree on formula $\varphi$. We will proceed in three steps: from partitions for $\phi$ and $\psi$ we will successively construct a partition for $\mathrm{E} \phi \mathbf{U} \psi$ and $\mathbf{A} \phi \mathbf{U} \psi$, then for $\mathrm{E} \phi \mathbf{U}_{\sim c} \psi$ and finally for $\mathrm{A} \phi \mathrm{U}_{\sim c} \psi$.

The first step is achieved using the following lemma, which applies the construction of a finite and definable partition (the so-called suffix-partition) correct w.r.t. bisimulation (see Theorem 5), and thus for CTL-formulae [AHLP00,HMR05].

Lemma 18. Let $\mathcal{P}_{\phi}\left(\right.$ resp. $\left.\mathcal{P}_{\psi}\right)$ be a partition for $\phi$ (resp. $\left.\psi\right)$. The partition $\operatorname{Suf}\left(\mathcal{P}_{\phi} \sqcap \mathcal{P}_{\psi}\right)$ is a time-abstract bisimulation and is a partition for formula $\mathrm{E} \phi \mathrm{U} \psi$. That is, if $P$ is a piece of $\operatorname{Suf}\left(\mathcal{P}_{\phi} \sqcap \mathcal{P}_{\psi}\right)$, then all states $(q, y)$ with $y \in P$ either satisfy $\mathrm{E} \phi \mathrm{U} \psi$ or do not satisfy this formula. The same holds for $\mathrm{A} \phi \mathbf{U} \psi$.

The second step, the construction of a (finite and definable) partition respecting $\mathrm{E} \phi \mathrm{U}_{\sim_{c}} \psi$, is more involved.

Let us denote by $\mathcal{P}$ the partition $\operatorname{Suf}\left(\mathcal{P}_{\phi} \sqcap \mathcal{P}_{\psi}\right)$. Let $q$ and $q^{\prime}$ be two locations of $\mathcal{H}$. Let $P$ and $P^{\prime}$ be two pieces of $\mathcal{P}$. We give formulae which define the set of possible costs of paths from a state (or a piece of $\mathcal{P}$ ) to another one:

$-\theta_{(q, P) \rightarrow\left(q^{\prime}, P^{\prime}\right)}^{\phi \vee \psi}(c, y)$ expresses that it is possible to go from some $(q, y)$ with $y \in P$ to some $\left(q^{\prime}, y^{\prime}\right)$ with $y^{\prime} \in P^{\prime}$ by a continuous step followed by a discrete action, with cost $c$, and always satisfying $\phi \vee \psi$.

$-\theta_{\left(q, P \rightsquigarrow P^{\prime}\right)}^{\phi \vee \psi}(c, y)$ expresses that it is possible to go from $(q, y)$ with $y \in P$ to some $\left(q, y^{\prime}\right)$ with $y^{\prime} \in P^{\prime}$ by a continuous step (and no discrete action), with cost $c$, and always satisfying $\phi \vee \psi$.

From the two previous formulae, we define the following definable sets:

$$
\begin{gathered}
\kappa_{(q, P) \rightarrow\left(q^{\prime}, P^{\prime}\right)}^{\phi \vee \psi}=\left\{c \in M^{+} \mid \exists y \in P \text { s.t. } \theta_{(q, P) \rightarrow\left(q^{\prime}, P^{\prime}\right)}^{\phi \vee \psi}(c, y)\right\} ; \\
\kappa_{\left(q, P \rightsquigarrow P^{\prime}\right)}^{\phi \vee \psi}=\left\{c \in M^{+} \mid \exists y \in P \text { s.t. } \theta_{\left(q, P \rightsquigarrow P^{\prime}\right)}^{\phi \vee \psi}(c, y)\right\} ; \\
\lambda_{(q, P) \rightarrow\left(q^{\prime}, P^{\prime}\right)}^{\phi \vee \psi}(y)=\left\{c \in M^{+} \mid \theta_{(q, P) \rightarrow\left(q^{\prime}, P^{\prime}\right)}^{\phi \vee \psi}(c, y)\right\} ; \\
\lambda_{\left(q, P \rightsquigarrow P^{\prime}\right)}^{\phi \vee \psi}(y)=\left\{c \in M^{+} \mid \theta_{\left(q, P \rightsquigarrow P^{\prime}\right)}^{\phi \vee \psi}(c, y)\right\} .
\end{gathered}
$$

We then construct a finite graph which abstracts away all dynamical parts of $\mathcal{H}$ and which will be restricted to parts of $\mathcal{H}$ which satisfy formula $\mathrm{E} \phi \mathrm{U} \psi$. Each edge of this graph will be labeled with a weight (indeed a definable set) which will represent the set of costs of all paths in $\mathcal{H}$ witnessing formula $\mathrm{E} \phi \mathrm{U} \psi$. More formally, we construct a (definable) weighted finite graph $\mathcal{G}_{\phi, \psi}=(V, E)$ as follows: 
- its set of vertices $V$ is

$$
\{(q, P),(q, P \text {, init }) \mid(q, P) \models \phi \vee \psi\}^{8} \cup \quad\{(q, P, \text { final }) \mid(q, P) \models \psi\}
$$

- its set of edges $E$ is

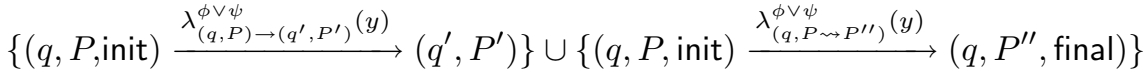

$$
\begin{aligned}
& \cup\{(q, P \text {, init }) \stackrel{[0]}{\longrightarrow}(q, P, \text { final })\} \cup\left\{(q, P) \stackrel{\kappa_{(q, P) \rightarrow\left(q^{\prime}, P^{\prime}\right)}^{\phi \vee \psi}}{\longrightarrow}\left(q^{\prime}, P^{\prime}\right)\right\} \\
& \cup\left\{(q, P) \stackrel{\kappa_{(q, P) \rightarrow\left(q^{\prime}, P^{\prime}\right)}^{\phi \vee \psi}}{\longrightarrow}\left(q^{\prime}, P^{\prime}, \text { final }\right)\right\} \cup\left\{(q, P) \stackrel{\kappa_{\left(q, P \rightsquigarrow P^{\prime \prime}\right)}^{\phi \vee \psi}}{\longrightarrow}\left(q, P^{\prime \prime} \text {, final }\right)\right\}
\end{aligned}
$$

Let $\varrho=\left(q_{0}, P_{0}\right.$, init $) \stackrel{\lambda(y)}{\longrightarrow}\left(q_{1}, P_{1}\right) \stackrel{\kappa_{1}}{\longrightarrow} \ldots \stackrel{\kappa_{n}}{\longrightarrow}\left(q_{n}, P_{n}\right.$, final $)$ be a finite path of $\mathcal{G}_{\phi, \psi}$. Then we define $K_{\varrho}(y)$ to be the set of all possible costs of the path $\varrho$ : $K_{\varrho}(y)=\left\{\lambda(y)+c_{2}+\cdots+c_{n} \mid c_{i} \in \kappa_{i}\right\}$.

The following proposition shows that the graph $\mathcal{G}_{\phi, \psi}$ can be used to modelcheck the formula $\mathrm{E} \phi \mathrm{U}_{\sim_{c}} \psi$.

Proposition 19. Let $q_{0} \in Q, P_{0} \in \mathcal{P}$ and $y_{0} \in P_{0}$. Then, $\left(q_{0}, y_{0}\right) \models \mathrm{E} \phi \mathrm{U}_{\sim_{c}} \psi$ iff there exists a path $\varrho=\left(q_{0}, P_{0}\right.$, init $) \stackrel{\lambda(y)}{\longrightarrow}\left(q_{1}, P_{1}\right) \stackrel{\kappa_{1}}{\longrightarrow} \ldots \stackrel{\kappa_{n}}{\longrightarrow}\left(q_{n}, P_{n}\right.$, final $)$ in $\mathcal{G}_{\phi, \psi}$, there exists $\zeta \in M$ such that $\zeta \in K_{\varrho}\left(y_{0}\right)$ and $\zeta \sim c$.

We prove that, under the Archimedian hypothesis, we can bound the length of witnessing paths in the graph $\mathcal{G}_{\phi, \psi}$. This hypothesis has not been used yet, as everything holds without it, but is required by the next proposition. For every $(m, c) \in M^{2}$ such that $m \neq 0$, we note $\left[\frac{c}{m}\right]$ the smallest integer $k$ such that $k \cdot m>c$

Proposition 20. We assume that $\left(q_{0}, y_{0}\right) \models \mathrm{E} \phi \mathrm{U}_{\sim_{c}} \psi$, and we define $N=2+$ $(|Q| \cdot|\mathcal{P}|+1) \cdot\left(\left[\frac{c}{m}\right]+2\right)$ where $m$ is the smallest positive constant defining an interval of some weight labelling the transitions of $\mathcal{G}_{\phi, \psi}$. Then there exists a path $\varrho=\left(q_{0}, P_{0}\right.$, init $) \stackrel{\lambda(y)}{\longrightarrow}\left(q_{1}, P_{1}\right) \stackrel{\kappa_{1}}{\longrightarrow} \ldots \stackrel{\kappa_{n}}{\longrightarrow}\left(q_{n}, P_{n}\right.$, final) in $\mathcal{G}_{\phi, \psi}$ such that $y_{0} \in P_{0}, n \leq N$, and there exists $\zeta \in M$ such that $\zeta \in K_{\varrho}\left(y_{0}\right)$ and $\zeta \sim c$.

Proof (Sketch). By Proposition 19, there exists a path $\varrho=\left(q_{0}, P_{0}\right.$, init $) \stackrel{\lambda(y)}{\longrightarrow}$ $\left(q_{1}, P_{1}\right) \stackrel{\kappa_{1}}{\longrightarrow} \ldots \stackrel{\kappa_{n}}{\longrightarrow}\left(q_{n}, P_{n}\right.$, final $)$ in $\mathcal{G}_{\phi, \psi}$ s.t. $\exists \zeta \in K_{\varrho}\left(y_{0}\right), \zeta \sim c$. From $\varrho$, we will construct a run of length smaller than $N$ which witnesses the formula.

By o-minimality, each $\kappa_{i}$ is a finite union of intervals. We first choose accurately one such interval per $\kappa_{i}$ and obtain a realisation of $\varrho$ noted $\varrho[\mathcal{I}]$, so that the accumulated union of all intervals along $\varrho[\mathcal{I}]$ contains $\zeta$ such that $\zeta \sim c$. We call [0]-cycle a cycle labeled only by the interval [0]. We can suppose that there is no $[0]$-cycle in $\varrho[\mathcal{I}]$ otherwise we can remove this cycle and get a shorter path also witnessing the formula.

8 This is a notation misuse, but $(q, P) \models \phi \vee \psi$ means that for all $y \in P,(q, y) \models \phi \vee \psi$, which is equivalent to the property that $(q, y) \models \phi \vee \psi$ for some $y \in P$. 


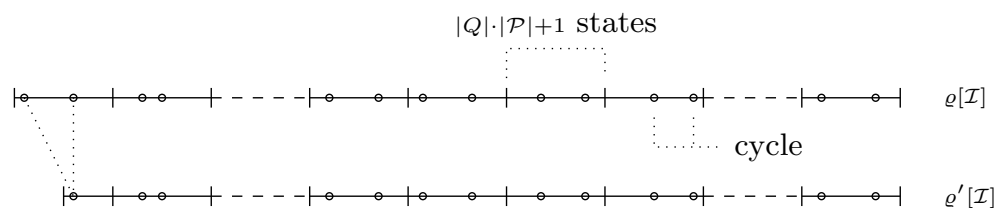

there may be [0]-cycles

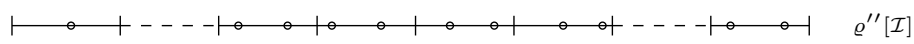

[0]-cycles have been removed

Fig. 2. The construction in the proof of Proposition 20

If the length of $\varrho[\mathcal{I}]$ is shorter than $N$ then we are done. Otherwise $\varrho$ contains at least $\left(\left[\frac{c}{m}\right]+2\right)$ simple cycles (see Fig. 2), each of which is labeled by an (accumulated) interval whose right bound is greater than $m$. Thus, $K_{\varrho[\mathcal{I}]}\left(y_{0}\right)=$ $\langle a, b\rangle^{9}$ with $b \geq c+2 m$. By hypothesis, there exists $\zeta \in\langle a, b\rangle$ such that $\zeta \sim c$.

We then remove the first cycle of the run and remove [0]-cycles that can have been potentially created (see Fig. 2 ). We obtain $\varrho^{\prime \prime}[\mathcal{I}]$ a strictly smaller run. Note that $K_{\varrho^{\prime \prime}[\mathcal{I}]}\left(y_{0}\right)$ might be different from $K_{\varrho[\mathcal{I}]}\left(y_{0}\right)$; nevertheless $\varrho^{\prime \prime}[\mathcal{I}]$ still has at least $\left[\frac{c}{m}\right]$ simple cycles so $K_{\varrho^{\prime \prime}[\mathcal{I}]}\left(y_{0}\right)=\left\langle a^{\prime}, b^{\prime}\right\rangle$ with $b^{\prime}>c$ (as previously). It proves that there exists $\zeta \in K_{\varrho^{\prime \prime}[\mathcal{I}]}\left(y_{0}\right)$ with $\zeta \sim c$ (since $c<b^{\prime} \leq b$ and $a^{\prime} \leq a$ ). We iterate this process until we find a run smaller than $N$ with this property.

Applying the previous proposition, we can build a first-order formula which checks if a given state $(q, y)$ satisfies the WCTL formula $\mathrm{E} \phi \mathrm{U}_{\sim c} \psi$, and we thus get the following corollary.

Corollary 21. Let $\mathcal{M}=\langle M,+, 0,1,<, \ldots\rangle$ be an o-minimal structure such that $\mathcal{M}$ is Archimedian and $\operatorname{Th}(\mathcal{M})$ is decidable. Let $\mathcal{H}$ be an $\mathcal{M}$-weighted hybrid system and $\phi$ and $\psi$ be two WCTL formulae. Assume we have built two finite and definable partitions $\mathcal{P}_{\phi}$ and $\mathcal{P}_{\psi}$ for $\phi$ and $\psi$, then we can compute a definable finite partition correct for the formula $\mathrm{E} \phi \mathrm{U}_{\sim c} \psi$.

We do not enter into the details of the third step. However, the construction of the partition respecting $\mathrm{A} \phi \mathrm{U}_{\sim c} \psi$ (from the partition $\mathcal{P}_{\phi}$ and $\mathcal{P}_{\psi}$ ) shares ideas with the construction of the partition respecting $\mathrm{E} \phi \mathrm{U}_{\sim c} \psi$ that we have described. In particular, the idea exploited in the proof of Proposition 20 plays an important role.

\section{References}

[ABM04] Rajeev Alur, Mikhail Bernadsky, and Parthasarathy Madhusudan. Optimal reachability for weighted timed games. In ICALP'04: Automata, Languages, and Programming, vol. 3142 of LNCS, pp. 122-133. Springer, 2004.

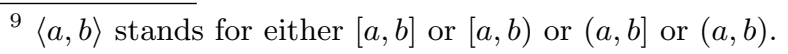


[AD94] Rajeev Alur and David Dill. A theory of timed automata. Theoretical Computer Science, 126(2):183-235, 1994.

[AHLP00] Rajeev Alur, Thomas A. Henzinger, Gerardo Lafferriere, and George J. Pappas. Discrete abstractions of hybrid systems. Proc. of the IEEE, 88:971984, 2000.

[ALP01] Rajeev Alur, Salvatore La Torre, and George J. Pappas. Optimal paths in weighted timed automata. In HSCC'01: Hybrid Systems, Computation and Control, vol. 2034 of LNCS, pp. 49-62. Springer, 2001.

[BBBR06] Patricia Bouyer, Thomas Brihaye, Véronique Bruyère, and Jean-François Raskin. On the optimal reachability problem. Submitted, 2006.

[BBC06] Patricia Bouyer, Thomas Brihaye, and Fabrice Chevalier. Control in ominimal hybrid systems. In LICS'06: Logic in Computer Science, pp. 367378. IEEE Computer Society Press, 2006.

[BBL04] Patricia Bouyer, Ed Brinksma, and Kim G. Larsen. Staying alive as cheaply as possible. In HSCC'04: Hybrid Systems, Computation and Control, vol. 2993 of LNCS, pp. 203-218. Springer, 2004.

[BBM06] Patricia Bouyer, Thomas Brihaye, and Nicolas Markey. Improved undecidability results on weighted timed automata. Information Processing Letters, 98(5):188-194, 2006.

[BBR04] Thomas Brihaye, Véronique Bruyère, and Jean-François Raskin. Modelchecking for weighted timed automata. In FORMATS-FTRTFT'04: Formal Modelling and Analysis of Timed Systems and Formal Techniques in RealTime and Fault-Tolerant Systems, vol. 3253 of LNCS, pp. 277-292. Springer, 2004.

[BBR05] Thomas Brihaye, Véronique Bruyère, and Jean-François Raskin. On optimal timed strategies. In FORMATS'05: Formal Modelling and Analysis of Timed Systems, vol. 3829 of LNCS, pp. 49-64. Springer, 2005.

[BBR06] Thomas Brihaye, Véronique Bruyère, and Jean-François Raskin. On modelchecking timed automata with stopwatch observers. Information and Computation, 204(3):408-433, 2006.

[BCFL04] Patricia Bouyer, Franck Cassez, Emmanuel Fleury, and Kim G. Larsen. Optimal strategies in priced timed game automata. In FSTTCS'04: Foundations of Software Technology and Theoretical Computer Science, vol. 3328 of LNCS, pp. 148-160. Springer, 2004.

$\left[\mathrm{BFH}^{+} 01\right]$ Gerd Behrmann, Ansgar Fehnker, Thomas Hune, Kim G. Larsen, Paul Pettersson, Judi Romijn, and Frits Vaandrager. Minimum-cost reachability for priced timed automata. In HSCC'01: Hybrid Systems, Computation and Control, vol. 2034 of LNCS, pp. 147-161. Springer, 2001.

[BLM07] Patricia Bouyer, Kim G. Larsen, and Nicolas Markey. Model-checking oneclock priced timed automata. In FoSSaCS'07: Foundations of Software Science and Computation Structures, vol. 4423 of LNCS, pp. 108-122. Springer, 2007.

[BLMR06] Patricia Bouyer, Kim G. Larsen, Nicolas Markey, and Jacob Illum Rasmussen. Almost optimal strategies in one-clock priced timed automata. In FSTTCS'06: Fundations of Software Technology and Theoretical Computer Science, vol. 4337 of LNCS, pp. 346-357. Springer, 2006.

[BM05] Thomas Brihaye and Christian Michaux. On the expressiveness and decidability of o-minimal hybrid systems. Journal of Complexity, 21(4):447-478, 2005. 
[BMRT04] Thomas Brihaye, Christian Michaux, Cédric Rivière, and Christophe Troestler. On o-minimal hybrid systems. In HSCC'04: Hybrid Systems, Computation and Control, vol. 2993 of LNCS, pp. 219-233. Springer, 2004.

[Bri06a] Thomas Brihaye. Verification and Control of O-Minimal Hybrid Systems and Weighted Timed Automata. Thèse de doctorat, Université MonsHainaut, Belgium, 2006.

[Bri06b] Thomas Brihaye. Words and bisimulation of dynamical systems. Journal of Automata, Languages and Combinatorics, 2006. To appear.

[cor06] Uppaal Cora, 2006. http://www.cs.aau.dk/〜behrmann/cora/.

[Dav99] Jennifer M. Davoren. Topologies, continuity and bisimulations. Theoretical Informatics and Applications, 33(4-5):357-382, 1999.

[Gen05] Raffaella Gentilini. Reachability problems on extended o-minimal hybrid automata. In FORMATS'05: Formal Modeling and Analysis of Timed Systems, vol. 3829 of $L N C S$, pp. 162-176. Springer, 2005.

[Hen95] Thomas A. Henzinger. Hybrid automata with finite bisimulations. In ICALP'95: Automata, Languages, and Programming, vol. 944 of LNCS, pp. 324-335. Springer-Verlag, 1995.

[Hen96] Thomas A. Henzinger. The theory of hybrid automata. In LICS'96: Logic in Computer Science, pp. 278-292. IEEE Computer Society Press, 1996.

[HMR05] Thomas A. Henzinger, Rupak Majumdar, and Jean-François Raskin. A classification of symbolic transition systems. ACM Transactions on Computational Logic, 6(1):1-32, 2005.

[Hod97] Wilfrid Hodges. A Shorter Model Theory. Cambridge University Press, 1997.

[KV06] Margarita V. Korovina and Nicolai Vorobjov. Upper and lower bounds on sizes of finite bisimulations of Pfaffian hybrid systems. In $C i E$, vol. 3988 of Lecture Notes in Computer Science, pp. 267-276. Springer, 2006.

[LMM02] Salvatore La Torre, Supratik Mukhopadhyay, and Aniello Murano. Optimalreachability and control for acyclic weighted timed automata. In TCS'02: Theoretical Computer Science, vol. 223 of IFIP Conf. Proc., pp. 485-497. Kluwer, 2002.

[LPS00] Gerardo Lafferriere, George J. Pappas, and Shankar Sastry. O-minimal hybrid systems. Mathematics of Control, Signals, and Systems, 13(1):1-21, 2000. Appeared as a preprint in 1998.

[PS86] Anand Pillay and Charles Steinhorn. Definable sets in ordered structures. Transactions of the American Mathematical Society, 295(2):565-592, 1986.

[Ras99] Jean-François Raskin. Logics, Automata and Classical Theories for Deciding Real-Time. Thèse de doctorat, Université Namur, Belgium, 1999.

[vdD98] Lou van den Dries. Tame Topology and O-Minimal Structures, vol. 248 of London Mathematical Society Lecture Note Series. Cambridge University Press, 1998.

[Wil96] Alex J. Wilkie. Model completeness results for expansions of the ordered field of real numbers by restricted Pfaffian functions and the exponential function. Journal of the AMS, 9(4):1051-1094, 1996. 